\title{
Study protocol: a randomised non-inferiority trial using interactive virtual presence to remotely assist parents with child restraint installations
}

\author{
David C Schwebel 자, 'Jennifer Morag MacKay, ${ }^{2}$ David Redden ${ }^{3}$
}

${ }^{1}$ Psychology, University of Alabama at Birmingham, Birmingham, Alabama, USA ${ }^{2}$ Safe Kids Worldwide, Washington, District of Columbia, USA

${ }^{3}$ Biostatistics, University of Alabama at Birmingham, Birmingham, Alabama, USA

\section{Correspondence to} Dr David C Schwebel, Psychology, University of Alabama at Birmingham, Birmingham, AL 35294, USA; schwebel@uab.edu

Received 2 September 2019 Revised 8 October 2019 Accepted 14 October 2019 Published Online First 14 November 2019

\section{Check for updates}

(c) Author(s) (or their employer(s)) 2020. No commercial re-use. See rights and permissions. Published by BMJ.

To cite: Schwebel DC, Mackay JM, Redden D. Inj Prev 2020;26:289-294.

\begin{abstract}
Background Motor vehicle crashes are the thirdleading cause of death to American children aged 1-5 years. When installed correctly, child restraints (car seats) reduce risk of serious injury and death. However, most restraints are installed incorrectly. The current gold standard for correct installation is systematic car seat checks, where certified technicians help parents, but car seat checks are highly underused due to barriers in access, scheduling and resources.
\end{abstract}

Methods The present study protocol describes plans to evaluate use of interactive virtual presence technology (interactive merged reality) - joint, simultaneous remote verbal and visual interaction and exposure to the same 3D stimuli-to assist remotely located parents installing child restraints. If effective, this technology could supplement or replace in-person checks and revolutionise how government, industry and non-profits help parents install child restraints properly. Building from preliminary studies, we propose a randomised non-inferiority trial to evaluate whether parents who install child restraints while communicating with remote expert technicians via interactive virtual presence on their smartphones achieve installations and learning not inferior in safety to parents who install restraints with on-site technicians. We will randomly assign 1476 caregivers at 7 US sites to install child restraints either via interactive virtual presence or live technicians. Correctness of installation will be assessed using objective checklists, both following installation and again 4 months later.

Conclusion We aim to demonstrate that child restraint installation is accurate (>90\% correct) when conducted remotely, that such installations are not inferior to installation accuracy with live experts and that parents learn and retain information about child restraint installation.

Motor vehicle crashes are the third-leading cause of death for American children aged 1-5 years. ${ }^{1}$ Morbidity rates far exceed mortality and impact public health tremendously. In 2017, over 500 American children aged 5 years and under were killed in motor vehicle crashes and over 37000 others were injured seriously enough to visit an emergency department. ${ }^{1}$ The estimated societal financial burden from motor vehicle injuries to children aged 5 years and younger in the USA exceeds 1.4 billion dollars. ${ }^{1}$

Child restraint systems (also called 'restraints' and 'car seats') reduce risk of serious injury and death to infants and young children roughly threefold, but are most effective when installed correctly. ${ }^{2-4}$ Unfortunately, multiple studies suggest a large portion of child restraints, usually in the 70\%-100\% range, are installed incorrectly. ${ }^{5-10}$ Although incorrect installation of restraints is preferred over lack of restraint, incorrect installation results in death or more serious injuries than might have occurred with proper installation and proper securing of children into the seats. ${ }^{1112}$

It is unclear exactly why child restraints are installed incorrectly with such frequency. ${ }^{13}$ One factor may be that manufacturer instruction manuals are difficult to comprehend or are not reviewed carefully by parents. Research suggests that manuals are written at a reading level that exceeds that of many consumers. ${ }^{14}$ Another explanation is the physical demands required to complete the task of installing a child restraint. Ergonomic research documents the fact that proper installation of child restraints requires some degree of strength and agility, especially if the installer is unfamiliar with optimal installation strategies. ${ }^{15}$ A third factor is that installation is simply very difficult. Correct installation often requires assistance from an expert. Several studies suggest hands-on individualised training of child restraint installation is effective and reduces errors significantly compared with use of the manufacturer manual alone. ${ }^{41617}$ In fact, installation and training from a Child Passenger Safety Technician ('technician' or 'certified technician') currently certified by an accredited body such as Safe Kids Worldwide (SKW) is considered the best strategy for correct installation of child restraints. ${ }^{18}$

\section{INCREASING THE RATE OF CORRECTLY INSTALLED CHILD RESTRAINTS}

Individualised one-on-one installation of child restraints is considered the gold standard for child safety, ${ }^{18}$ but available evidence concerning incorrect installation rates (70\%-100\% range) and appointments at certified car-seat inspection stations and car seat checks (also called check-up events) suggest that it is practiced quite infrequently. In Florida, for example, certified technicians at car seat checks installed about 15000 child restraints in the year $2015 .{ }^{19}$ In that same year, there were over 224000 live births in Florida, ${ }^{20}$ implying far less than $5 \%$ of child restraints on the roadways in Florida were installed with assistance from a certified technician.

There are several barriers to use of car seat checks. ${ }^{18}$ One is convenience. Many families with 
young children live busy, chaotic and/or stressful lives and the task of obtaining, remembering and attending an appointment for a car seat check may fall low in the list of household priorities. Another is access. Organising and staffing car seat checks with certified technicians is expensive for government or nonprofit agencies to administer. Demand greatly outpaces supply in most locations, and access is particularly poor in rural and frontier areas of the country. The present study will evaluate whether we might overcome all these barriers through use of mobile interactive virtual presence that permits remotely located certified technicians to interact with parents to install child restraints in their vehicles at convenient times and places using a mobile smartphone.

\section{INTERACTIVE VIRTUAL PRESENCE}

Recent technological advances in interactive virtual presence-technology that integrates augmented and merged virtual reality to permit remote experts to train lay or professional individuals-suggests that it can be effective in a wide variety of tasks through joint exposure to 3D images and interactive simultaneous engagement with the object(s) of interest. For example, a remotely located machinist can help a labourer repair the electrical circuit on a complex piece of factory equipment without travelling on-site for repairs. Such training paradigms have been demonstrated effective in the medical and surgical fields, ${ }^{21-23}$ and in maintenance of industrial machinery. ${ }^{24}$

In technical language, interactive virtual presence refers to the opportunity for users to simultaneously engage in interactive visual, nonverbal and aural communication. It provides merged reality and concurrent virtual interaction. Users can instantly and simultaneously view and merge two real-time perspectives, offering opportunity for remote collaboration while interactively examining, pointing to, grasping, illustrating and discussing a video stream. Images can be frozen, drawn on, animated and viewed live. Interactive virtual presence can be delivered through a range of hardware platforms, including tablets and mobile smartphones.

In lay language, interactive virtual presence implies that users requiring help - in the case of our research, parents-may place their smartphone over a targeted area to allow the expert-in our case, a remotely located certified technician-to 'freeze' that image and then point to or grasp particular areas with their hands and/or with telestration tools like arrows and pointers located within the software while speaking. Thus, for example, if a parent was unsure where to connect a child restraint anchor, she could use her smartphone to show the technician the back seat of the car and request that the technician point with her finger to the location of the anchor. Similarly, a parent could direct his phone's camera towards a child restraint to verify it is installed well and the technician might notice a loose strap, draw a circle around that strap and request that the parent tighten it. Interactive virtual presence extends beyond traditional telemedicine and video chat, instead providing the opportunity to engage immersively in all tasks and activities that a live certified technician provides, but using technology from a remote location.

\section{THE COGNITIVE AND BEHAVIOURAL BASES OF CHILD RESTRAINT INSTALLATION}

Installing a child restraint properly is a challenging task that requires complex cognitive processing and logical thinking, some degree of physical strength and dexterity, patience and persistence and practice and training. Empirical research consistently demonstrates that parents are poor at the task when they engage in it without any resources or with only the manufacturer's instruction manual. ${ }^{416} 17$

The best alternative strategy to self-installation, and the recommendation of both paediatricians and traffic safety experts, is use of individualised instruction, education and assistance from a currently certified technician. ${ }^{18} 2526$ Recent evidence from a study of 291 parents, some of whom had recently worked with certified technicians to install their restraint, showed that parents who worked with technicians had 90\% fewer critical misuse errors than parents who had not worked with technicians. ${ }^{9}$ Interactions with technicians may also have some lasting effect over time. Among one sample of 47 parents, interaction with a certified technician yielded a significant drop in any misuse of the restraint on a 4-month follow-up (from $80 \%$ to $66 \%$ ), as well as a significant drop in 'critical' misuse (from 52\% to $40 \% ;^{3}$ see also Mirman et al. ${ }^{27}$ ). There are currently about 40000 nationally certified technicians in the USA, and they are concentrated primarily in larger cities.

\section{RESEARCH DESIGN}

Building from our preliminary research, ${ }^{28-30}$ we will conduct a randomised non-inferiority trial to test whether parents can accurately install child restraints based on advice and guidance received remotely from certified technicians using interactive virtual presence. We hypothesise that communicating this information to parents using interactive virtual presence will be not inferior to results obtained using the primary recommended strategy in the field, live one-on-one installation advice from a certified technician. Our trial will follow CONSORT 2010 guidelines, as extended for non-inferiority randomised trials. ${ }^{31}$

\section{Participants and sites}

A total of 1476 individuals who frequently drive children in their vehicles (henceforth, 'parents') will be recruited from the geographic footprints of 7 SKW centres spread geographically across the USA: Safe Kids Alabama/Children's Health System (based in Birmingham, Alabama, USA); Safe Kids California, Los Angeles/Los Angeles Children's Hospital (based in Los Angeles, California, USA); Safe Kids Charlotte/Mecklenburg/ Carolinas Medical Center (based in Charlotte, North Carolina, USA); Safe Kids Oklahoma/The Children's Center Rehabilitation Hospital (based in Oklahoma City, Oklahoma, USA); Safe Kids Greater Houston (based in Houston, Texas, USA); Safe Kids Vermont/University of Vermont Children's Hospital (based in Burlington, Vermont, USA) and Safe Kids Lower Columbia/Cowlitz County EMS and Trauma Care Council (based in Kelso, Washington, USA). All centres have large geographic footprints that they serve, and we will oversample recruitment of families from rural and underprivileged areas, who have higher documented risk of car seat misuse, ${ }^{32} 33$ at each site. We plan to recruit comparable numbers of families from each of the seven sites.

Across all sites together, we will stratify recruitment to achieve a sample that is about 50\% from rural locations, 35\% nonHispanic White and 65\% from racial and/or ethnic minority groups. All participants will own a vehicle and have a child who rides in a child restraint fastened with a harness. Booster seats, which are comparatively simple to install and use, will be excluded. The research will be conducted in both English and Spanish and all study instruments and guidelines will be translated to Spanish through standard translation and backtranslation strategies. 
Table 1 Self-report measures

\begin{tabular}{|c|c|c|}
\hline Measure & \# Items & Administration \\
\hline Demographics - Parent (age, gender, race, ethnicity, family income, education, relation to child) & 7 & Baseline \\
\hline Demographics —Child (age, gender, race, ethnicity, birth order) & 5 & Baseline \\
\hline Attitudes/Beliefs about restraints: Is your restraint correctly installed? & 1 & Baseline, post, follow-up \\
\hline Attitudes/Beliefs about restraints: Can you install correctly? & 1 & Baseline, post, follow-up \\
\hline Attitudes/Beliefs about restraints: Is restraint installation difficult? & 1 & Baseline, post, follow-up \\
\hline Attitudes/Beliefs about restraints: Will your child be injured in crash? & 1 & Baseline, post, follow-up \\
\hline Restraint behaviours: Previous help from technicians & 1 & Baseline, post, follow-up \\
\hline Restraint behaviours: Use of instruction manual & 1 & Baseline, post, follow-up \\
\hline Restraint behaviours: Use of car seat (frequency) & 1 & Baseline, post, follow-up \\
\hline Perceived efficacy of training & 6 & Post, follow-up \\
\hline
\end{tabular}

\section{Protocol}

Participants will be recruited through targeted advertising, especially via social media. On expression of interest in the study, potential participants will be able to access a website to gather more information, communicate with the research team and/or consent to participate. Consenting will be conducted electronically and remotely, although study personnel will be available to answer questions from participants.

Following consent, parents will complete brief questionnaires via a secure internet portal to report demographic background and knowledge, attitudes, beliefs and behaviours about child restraint installations (tables 1 and 2). Demographic information will ensure appropriate stratification to achieve our desired sample and will also be evaluated as moderating or covarying factors secondary to our primary hypotheses. Items on attitudes, beliefs and behaviour surrounding child restraints will serve as covariates and also address secondary questions of interest. Knowledge items will serve as a secondary outcome measure.

Following completion of the online questionnaires, parents will schedule an initial research appointment at their home, business or public location. Appointment scheduling will be conducted electronically, with appointment times offered at a range of convenient hours, including weekends and evenings.

Once an appointment time and location is selected by a participant, a local trained researcher and child passenger safety (CPS) technician will be assigned to the research visit. All visits will be conducted by two individuals, a researcher (who is also certified as a CPS technician) and a technician. Initial research visits will last about 1 hour. The researcher will confirm informed consent and inspect and score the current restraint installation. Then, the researcher will inform the participant of their random assignment to a condition, either installation with a certified technician via interactive virtual presence or installation via the traditional live technician interaction. In cases where there is more than one restraint in the vehicle, the researcher will use a random number generator to select one of the restraints as the target for the research.

Those parents randomly assigned to the interactive virtual presence condition will be instructed how to connect to the interactive virtual presence programme, as described above, using their personal smartphone. On rare occasions when the participant does not have a smartphone or data service capacity at the location, the participant will be included as an unsuccessful car seat installation for intent-to-treat analyses and the on-site technician will complete standard restraint installation processes. Otherwise, participants will connect to the interactive virtual presence programme (no cost to consumers), view a brief online instructional video on using it and then connect remotely to a certified technician.

Ensuring ecological validity and pragmatic trial practice, the researcher and local technician will purposely 'stay away' through the remote installation process, allowing the parent and remote technician to engage via interactive virtual presence to install the restraint correctly in the vehicle. Remote technicians will guide the interaction following standard SKW practice for live in-person installations and parents will physically install and adjust the restraint into their own vehicle. We expect the immersive and telestration tools of interactive virtual presence will be used extensively to aid in correct installations.

Those parents assigned to the control condition, installation by a live technician, will engage in standard procedures at SKW car seat checks to install the target restraint into the vehicle. The live technician will not be the same individual as the researcher, although they will both be present at the same location during the research protocol.

Following installation in both conditions, the researcher, who will also have CPS technician certification, will evaluate the accuracy of the installation using objective coding sheets developed in our pilot research. ${ }^{28-30}$ While that inspection occurs, parents will complete a second brief questionnaire concerning attitudes, beliefs and knowledge about child restraint installation. We also will evaluate perceived efficacy about the interventions from parents in both groups.

Following all data collection, the on-site technician will ensure all restraints in the vehicle, both those used in the research and others in the vehicle, are properly installed. Parents will be reimbursed for their time. All technicians, including those on-site and working remotely with participants and the researcher, will be certified by the SKW National Child Passenger Safety Technician Certification programme.

Four months later, a second research visit will be scheduled. During this visit, three activities will occur: (1) recompletion of

Table 2 Outcome measures

\begin{tabular}{lll}
\hline Measure & \# Items & Administration \\
\hline Number of inspection points correctly installed & $29-35$, depending on car and restraint & Baseline, post, follow-up baseline, follow-up exit \\
Dichotomous measure: restraint installed correctly versus not & 1 & Baseline, post, follow-up baseline, follow-up exit \\
\hline Knowledge-Installing restraints & 5 & Baseline, post, follow-up \\
\hline
\end{tabular}


Figure 1 Outline of study protocol.

questionnaires to assess knowledge, attitudes and beliefs about child restraint installation, plus items concerning perceived efficacy of the intervention for both groups and on behaviour over the 4-month period (eg, Was the restraint removed and/ or adjusted for child growth and then reinstalled? By whom?); (2) conducted simultaneously to parent questionnaire completion, assessment of current restraint installation by the CPS technician-certified researcher using same objective coding sheets to assess current restraint installation accuracy after 4 months and (3) removal and reinstallation by the parent of the child restraint, without certified technician assistance and then assessment of parental installation using objective coding sheets to assess retention of learnt restraint installation processes.

The follow-up visit will therefore evaluate both groups' retention of parent learning, current restraint safety, current ability to safely install a restraint and follow-up evaluation of parental attitudes, beliefs and perceived efficacy of the intervention. At the completion of the follow-up visit, the certified technician will reinstall all restraints in the parent's vehicle using standard Safe Kids protocol and parents will be reimbursed for their time.

Figure 1 illustrates the study protocol graphically.

\section{Outcome measures}

We will consider two primary outcomes, both derived from inspections of child restraint installations using objective coding sheets: (1) total number of inspection points that are correctly installed, translated into a percentage to account for different points for different (eg, rear-facing versus forward-facing; seat belt versus Lower Anchors and Tethers for CHildren (LATCH)) installations and (2) dichotomous measure of whether the restraint was installed correctly versus not correctly (see table 2). Examples of inspection points include whether tether straps are twisted, whether the seat moves more than 1 inch to the left/right, whether seat belts are routed through the correct belt pathway and whether the carrying handle is in the correct position. Both outcomes will be collected at four time points: at baseline prior to any intervention, at post, immediately following the initial installation in both groups, at the start of the follow-up visit following 4 months of no active intervention by the research team and at follow-up after parent installation without technician assistance.

We also will consider results from the brief knowledge questionnaire, which was adopted from an existing instrument. ${ }^{34}$ Secondary and covariate measures considered in all analyses will include demographic traits of the participant (eg, gender, race, ethnicity, socio economic status (SES), research site) and restraint (eg, forward versus rear facing, LATCH versus seat belt installation), attitudes and beliefs about child restraints, perceived efficacy of the interventions and reported behaviours using restraints.

\section{STATISTICAL ANALYSIS PLAN AND POWER Descriptive statistics and covariates}

Descriptive statistics for participants randomised to each intervention will be summarised for each outcome using measures of central tendency (mean, median, proportion) and variability (variance, SD, range). Several covariates may impact the relation between the intervention and the outcome measures of interest, and they will be similarly summarised descriptively. These include demographics (age, gender, target child birth order, race, ethnicity, SES); study site; type of vehicle; type of child restraint (including forward-facing versus rear-facing) and attitudes, beliefs and behaviours surrounding child restraints. Because parents will be randomised to the interventions, we expect covariates to be balanced across intervention groups. We will assess balance across intervention groups and use covariates in the primary analyses if appropriate. We also will estimate interaction effects to determine whether the intervention is more or less effective among particular subgroups that are adequately represented in the sample.

\section{Primary analyses}

We have three specific aims. Specific Aim 1 is to identify how accurate parents are at installing child restraints using instructions from technicians via interactive virtual presence. We expect to find over $90 \%$ or greater of restraints are installed correctly in all respects. We also will investigate individual components of installation and expect over $90 \%$ of components, across participants, will be installed correctly. 95\% CIs for all of these outcomes will be calculated and presented graphically as a Forrest Plot with a reference line set at 0.90 . Identical analyses will be conducted for the group randomised to in-person installation.

Specific Aim 2 will test whether child restraint installation by interactive virtual presence achieves installation accuracy at a rate not inferior to installation with a live technician. Two models will be computed, one with a measure of installation accuracy (proportion of all child restraint installation components performed correctly) as the dependent variable and the other with the dichotomous outcome of correct $(100 \%$ correct installation across all components) versus incorrect installation $(<100 \%$ correct installation) as the dependent variable. Assuming that improvement is reflected by a positive value (ie, a higher post-training value is better), our primary model, based on installation accuracy, is:

$$
\mathrm{H}_{O}: \Delta_{\text {LIVE }}-\Delta_{\text {IVP }} \geq \delta \text { vs. } \mathrm{H}_{\mathrm{A}}: \Delta_{\mathrm{LIVE}}-\Delta_{\mathrm{IVP}}<\delta
$$

where $\delta$ is the non-inferiority margin and IVP stands for interactive virtual presence. We will assume a non-inferiority margin of 0.025 for the primary analysis (see power analysis below) and perform analysis of covariance to determine if the difference of mean installation accuracy scores between the two groups falls below the non-inferiority margin of 0.025 , after adjustment for the baseline measure of installation accuracy and other relevant covariates. Based on previous work, ${ }^{28} 29$ we do not anticipate 
non-normality of the continuous outcome variable of installation accuracy. However, we will assess normality using graphical techniques (normal probability plot, histograms) prior to analysis and will transform outcomes if appropriate. The one-sided non-inferiority test will be conducted using a Type I error of 0.025 .

Specific Aim 3 will test parent learning and retention, both through measures of installation accuracy (after the intervention, at start of follow-up visit and after parent reinstallation during follow-up visit) and through the brief knowledge questionnaire administered at post and at follow-up. Six models will be computed using strategies identical to those for Aim 2 and predicting the following dependent variables: (1) installation accuracy (proportion of restraint components performed correctly at start of 4-month follow-up visit); (2) dichotomous outcome of correct versus incorrect installation at start of follow-up visit; (3) installation accuracy after parental reinstallation during follow-up visit; (4) dichotomous outcome of correct versus incorrect installation after parental reinstallation during follow-up visit; (5) knowledge questionnaire during postvisit and (6) knowledge questionnaire during follow-up visit. Statistical analysis system will be used for all analyses.

\section{Power analysis and sample size justification}

To conclude non-inferiority of the interactive remote presence condition with $\geq 80 \%$ power, Type I error rate of 0.025 , a noninferiority margin of 0.025 and assuming a common SD of 0.10 (similar to that observed previously), ${ }^{28} 29$ we propose a sample size of 1476 . This estimate conservatively assumes $10 \%$ attrition based on a two-sample test of non-inferiority difference in means. We estimated $10 \%$ attrition based on previous experience in our laboratories and will use a wide range of strategies to reduce attrition, including: (1) collection of multiple phone and email addresses for participants at baseline to reach them for follow-up scheduling, (2) baseline collection of contact information for two close friends or family members to assist with contact at follow-up, (3) use of a diverse and multicultural research staff who connect culturally to a diverse and multicultural research participant pool, (4) provision of fair and appropriate financial incentives to reimburse study participants for their time in the study and (5) scheduling of research appointments at convenient times and places for research participants.

Each cell of table 3 presents the statistical power to declare non-inferiority between two means, as defined by the alternative hypothesis, assuming a common SD of 0.10 , a Type I error rate of 0.025 , a sample size per group of 664 , the non-inferiority margin $(\Delta)$ specified in the row and the different combinations of intervention means $(\mu 1, \mu 2)$ specified by the columns. As long as the difference between the two means is less than the assumed non-inferiority margin, the definition of non-inferiority is met and power can be calculated. However, as can be seen by each row of the table, as the difference in intervention means grows, statistical power will decrease.

Table 3 Statistical power assuming 664 persons randomised per group (total $n=1476$ with conservatively estimated $10 \%$ attrition)

\begin{tabular}{llllll}
\hline \multicolumn{5}{c}{ Combination of mean accuracy scores for intervention groups } \\
\hline$\Delta$ & $\mu 1=0.90$ & $\mu 1=0.90$ & $\mu 1=0.90$ & $\mu 1=0.90$ & $\mu 1=0.90$ \\
& $\mu 2=0.90$ & $\mu 2=0.8975$ & $\mu 2=0.895$ & $\mu 2=0.8925$ & $\mu 2=0.8900$ \\
0.020 & 0.954 & 0.890 & 0.780 & 0.625 & 0.445 \\
0.025 & 0.995 & 0.984 & 0.954 & 0.890 & 0.780 \\
0.030 & 0.999 & 0.999 & 0.995 & 0.984 & 0.954 \\
\hline
\end{tabular}

To illustrate interpretation of the table, if the mean installation accuracy of in-person instruction is 0.90 and the mean installation accuracy of remote instruction is 0.90 (ie, the two groups achieve equal accuracy), then 664 persons randomised to each group provides $99.5 \%$ power to declare non-inferiority of remote instruction to in-person instruction using a Type I error rate of 0.025 , a non-inferiority margin of 0.025 and a common SD of 0.10 . Similarly, if the mean installation accuracy of in-person instruction is 0.90 and the mean installation accuracy of remote instruction is 0.89 , the two means are not equal but the difference in means $(0.90-0.89=0.01)$ remains less than the declared non-inferiority margin $(0.025)$. Under this scenario, 664 persons randomised to each group provide $78 \%$ power to declare noninferiority of remote instruction to in-person instruction using a Type I error rate of 0.025 , a non-inferiority margin of 0.025 and a common SD of 0.10 .

\section{Randomisation}

Randomisation lists will be generated by the study biostatistician and stored electronically. A separate randomisation list will be generated for each site and all will use a randomly permuted block design to allow for balance among intervention arms as the research study progresses and to limit the ability of any research staff member from definitively knowing/predicting future assignment. Block sizes will range over all even numbers from 8 to 16 inclusive.

\section{LIMITATIONS}

Like all research, this study will have limitations. First, it is not possible logistically to arrange for the second research visit to be unannounced. Thus, parents will know when it is scheduled and might potentially install their child restraint more securely or more accurately than they typically keep it. This limitation should be present across both conditions, reducing the impact on primary hypotheses to some extent. Second, we will experience some attrition in our sample. We conducted our power analysis with a conservative estimate of $10 \%$ attrition and will implement several strategies to minimise attrition, but we recognise the inevitable loss of participants in any longitudinal study and will adjust as appropriate statistically through techniques like imputation of missing data and conduct of intent-to-treat analyses.

\section{LONG-TERM VISION FOR PUBLIC HEALTH}

If our study hypotheses prove true, use of interactive virtual presence could supplement or replace current practice for child restraint inspections by certified technicians. We envision the possibility of movement towards 'car seat installation centres' that use as a model the system of Poison Control Centers across the USA. Such centres offer 24 hours on-call expertise to parents (and others) concerned about possible child poisoning ${ }^{35}$ and could be replicated with a centralised Car Seat 'Call' Center.

The Car Seat Center we envision-pending achievement of hypothesised results from this study-would be staffed by certified technicians who provide remote assistance from a centralised location to anywhere in the region, country or world using interactive virtual presence software downloaded to consumers' smartphones. The 'centre' might be funded by government, industry (eg, restraint manufacturers) or non-profit agencies. Secondary objectives could be addressed also. For example, the centre could provide expertise for complex and unusual child restraint installations, including those for children with special needs and disabilities, for families who do not speak English and for unusual vehicle designs (eg, vintage or antique vehicles). 
Eventually the model might even be extended to other child safety objectives (eg, installing cabinet locks, checking smoke detectors, inspecting homes for safety) or other paediatric health initiatives (eg, choosing healthy snacks, diabetes management).

Contributors The study was conceived by DCS and JMM. All authors contributed to drafting and critically reviewing the manuscript, and all approved the final version of the manuscript.

Funding Research reported in this publication was supported by the Eunice Kennedy Shriver National Institute of Child Health \& Human Development of the National Institutes of Health under Award Number R01HD099131. The content is solely the responsibility of the authors and does not necessarily represent the official views of the National Institutes of Health.

Competing interests None declared.

Patient consent for publication Not required.

Provenance and peer review Not commissioned; externally peer reviewed.

ORCID iD

David C Schwebel http://orcid.org/0000-0002-2141-8970

\section{REFERENCES}

1 Centers for Disease Control and Prevention. Welcome to WISQARS, 2019. Available: https://www.cdc.gov/injury/wisqars/

2 Berg MD, Cook L, Corneli HM, et al. Effect of seating position and restraint use on injuries to children in motor vehicle crashes. Pediatrics 2000;105:831-5.

3 Burstein D, Zonfrillo MR, Baird J, et al. Child passenger safety technician consultation in the pediatric primary care setting. Clin Pediatr 2017;56:928-33.

4 Lane WG, Liu GC, Newlin E. The association between hands-on instruction and proper child safety seat installation. Pediatrics 2000;106:924-9.

5 Bachman SL, Salzman GA, Burke RV, et al. Observed child restraint misuse in a large, urban community: results from three years of inspection events. I Safety Res 2016;56:17-22.

6 Blair J, Perdios A, Babul S, et al. The appropriate and inappropriate use of child restraint seats in Manitoba. Int J Inj Contr Saf Promot 2008;15:151-6.

7 Brown J, Hatfield J, Du W, et al. The characteristics of incorrect restraint use among children traveling in cars in New South Wales, Australia. Traffic Inj Prev 2010:11:391-8.

8 Duchossois GP, Nance ML, Wiebe DJ. Evaluation of child safety seat checkpoint events. Acc Anal Prev 2008;40:1908-12.

9 Hoffman BD, Gallardo AR, Carlson KF. Unsafe from the start: serious misuse of car safety seats at newborn discharge. J Pediatr 2016;171:48-54.

10 Koppel S, Charlton JL. Child restraint system misuse and/or inappropriate use in Australia. Traffic Inj Prev 2009;10:302-7.

11 Beringer-Brown C, Pearce J, Rush C. Child restraint misuse: a case example and strategies for injury prevention. Accid Emerg Nurs 2005;13:82-6.

12 Lesire P, Cuny S, Alonzo F, et al. Misuse of child restraint systems in crash situations - danger and possible consequences. Annual Proc Assoc Adv Automot Med 2007:51:207-22.
13 Hall A, Ho C, Keay L, et al. Barriers to correct child restraint use: a qualitative study of child restraint users and their needs. Saf Sci 2018:109:186-94.

14 Wegner MV, Girasek DC. How readable are child safety seat installation instructions? Pediatrics 2003:111:588-91.

15 Brown SHM, Grondin DE, Potvin JR. Strength limitations to proper child safety seat installation: implications for child safety. Appl Ergon 2009;40:617-21.

16 Brown J, Finch CF, Hatfield J, et al. Child restraint fitting stations reduce incorrect restraint use among child occupants. Acc Anal Prev 2011;43:1128-33.

17 Tessier K. Effectiveness of hands-on education for correct child restraint use by parents. Acc Anal Prev 2010;42:1041-7.

18 Will KE, Geller ES. Increasing the safety of children's vehicle travel: from effective risk communication to behavior change. J Safety Res 2004;35:263-74.

19 Florida Occupant Protection Resource Center. Florida OPRC year 5 summary, 2016. Available: http://www.floridaoprc.ce.ufl.edu/oprc/default.asp

20 Florida Department of Health. Florida vital statistics annual report 2015, 2016. Available: http://www.flpublichealth.com/vSBOOK/pdf/2015/Births.pdf

21 Barsom EZ, Graafland M, Schijven MP. Systematic review on the effectiveness of augmented reality applications in medical training. Surg Endosc 2016;30:4174-83.

22 Davis MC, Can DD, Pindrik J, et al. Virtual interactive presence in global surgical education: international collaboration through augmented reality. World Neurosurg 2016;86:103-11.

23 Silva JNA, Southworth M, Raptis C, et al. Emerging Applications of Virtual Reality in Cardiovascular Medicine. JACC: Basic to Translational Science 2018;3:420-30.

24 Gavish N, Gutiérrez T, Webel S, et al. Evaluating virtual reality and augmented reality training for industrial maintenance and assembly tasks. Interactive Learning Environments 2015;23:778-98.

25 Arbogast KB, Durbin DR, Morris SD. Assessing child restraint misuse by parental survey. Inj Prev 2000:6:145-7.

26 Biagioli F. Proper use of child safety seats. Am Fam Physician 2002;65:2085-90.

27 Mirman JH, Curry AE, Winston FK, et al. Parental influence on driver licensure in adolescence: a randomized controlled trial. Health Psychology 2017;36:245-54.

28 Schwebel DC, Johnston A, Rouse J. Teaching infant car seat installation via interactive visual presence: an experimental trial. Traffic Inj Prev 2017;18:188-92.

29 Schwebel DC, Tillman MA, Crew M, et al. Using interactive virtual presence to support accurate installation of child restraints: efficacy and parental perceptions. J Safety Res 2017;62:235-43

30 Swanson M, MacKay M, Yu S, et al. Supporting caregiver use of child restraints in rural communities via interactive virtual presence. Health Educ Behav. In Press.

31 Piaggio G, Elbourne DR, Pocock SJ, et al. Reporting of noninferiority and equivalence randomized trials: extension of the CONSORT 2010 statement. JAMA 2012;308:2594-604.

32 Hafner JW, Kok SJ, Wang H, et al. Child passenger restraint system misuse in rural versus urban children: a multisite case-control study. Ped Emerg Care 2017;33:663-9.

33 Macy ML, Cunningham RM, Resnicow K, et al. Disparities in age-appropriate child passenger restraint use among children aged 1 to 12 years. Pediatrics 2014:133:262-71.

34 MacKay M, Steel A, Walker L, et al. Child passenger safety: impact of buckle up inspections on caregiver knowledge, confidence and skill. Washington, DC: Safe Kids Worldwide, 2017

35 Gummin DD, Mowry JB, Spyker DA, et al. 2017 annual report of the American association of poison control centers' national poison data system (NPDS): 35 th annual report. Clin Toxicol 2018;56:1213-415. 